\title{
Numerical and experimental determination of in-structure temperature profiles
}

\author{
Marek Zozulák, Dušan Katunský \\ Technical University of Košice, Slovakia \\ Civil Engineering Faculty, Institute of Architectural Engineering \\ e-mail: marek.zozulak@tuke.sk
}

\begin{abstract}
When building physics simulations are done initial conditions express the actual hygrothermal state of building envelope. For the temperature field simulations initial condition is represented by the initial temperature in the body profile at the start of heat transfer. In-structure temperature varies quickly so temperature initial conditions are often neglected. Nevertheless in specific cases initial conditions have to be taken into an account even when simple temperature field simulations are done. The contribution shows various temperature initial conditions determination for insulated construction of outdoor test cell. Comparison of measured and calculated temperature profiles in structure shows correctness of start-up pre-calculation initial condition consideration.
\end{abstract}

Key words: start-up pre-calculation, initial temperature, temperature profile, measurement, simulation

\section{Introduction}

To undertake simulation input data including material properties, initial condition and boundary settings are input as constants, analytical expressions or interpolation files [1]. At the room level a steady state simulation could calculates the indoor airflow field, temperature and humidity distributions, and these values are set as the initial values of the transient analysis [2]. Correct estimation of the initial conditions can have significant impact on the predicted solutions also at the whole building level [3]. Therefore, when performing the analysis of building structures in simulation tools it is necessary to enter the initial temperature and moisture content. Temperature initial condition is considered as rapidly changing factor usually considered as a constant temperature of material built into the building structure. Nevertheless, more of simulation tools support the possibility of using the initial temperature condition as course of measured or calculated values, for example by making start-up pre-calculation. The aim is to show the importance of considering start-up pre-calculation in the process of simulation and its impact on the course of temperature. Effect of the pre-calculation on the simulation results is confronted with the measured temperatures characteristic within parts of the experimental wall of the outdoor test cells. The 
result will determine its consideration need according to the length of the calculation. Validation of the simulation is a valuable tool for controlling the accuracy of numerical experiments. Outdoor test cells or laboratory climate chambers equipment are appropriate instruments. Our institution has already conducted the similar research [4]. Structures fullscale state monitoring in non-stationary boundary conditions is a current trend of building physics researches [5]. Appropriate devices are outdoor on-site test cells for energy and hygrothermal assessment of structures implemented in Dübendorf, Glasgow, Cottbus, Limelette, Almeria, Espoo, Delft etc., since 1993 [6 \& 7]. In order to work with measured data local climatic data pertaining to outdoor test cells placement should be collected. In such a case it is helpful to use data from a nearby weather station [8].

\section{Methodology}

\subsection{Transient numerical simulation of temperature field}

Heat transfer in time is described by the heat diffusion equation (1). For balancing of temperature in the structure thermal diffusivity is critical. Transient calculation is made in commercial Physibel software, module BISTRA. The energy balance method is used to set up a system of linear equations. The system is solved using the Crank-Nicolson finite difference method. This method meets the criteria of the standard STN EN ISO 10211 Annex A, for software computing methods [9].

$$
\operatorname{div}(\lambda \operatorname{grad} T)+Z_{q}=c . \rho \cdot \frac{\partial T}{\partial t}
$$

where: $\lambda(\mathrm{W} / \mathrm{m} . \mathrm{K})$ thermal conductivity, $\rho\left(\mathrm{kg} / \mathrm{m}^{3}\right)$ bulk density, $c(\mathrm{~J} /(\mathrm{kg} . \mathrm{K}))$ specific heat, $q$ $\left(\mathrm{W} / \mathrm{m}^{2}\right)$ heat flux, $Z_{q}\left(\mathrm{~W} / \mathrm{m}^{3}\right)$ heat source, $T(\mathrm{~K})$ thermodynamic temperature, $t(\mathrm{~h})$ time.

\subsection{Methodology of determination of temperature initial conditions}

As the initial temperature before the start of calculation (before $t=0 \mathrm{~h}$ ) is considered:

- in time $t=0$ steady state temperature is calculated from actual ambient temperatures, no start-up pre-calculation;

- $\quad$ start-up pre-calculation used (calculation before $t=0 \mathrm{~h}$ duration of 1 day);

- measured value of temperature over the chosen time period.

\subsection{Experimental setup}

The various opaque and transparent envelope parts were monitored - temperature and relative humidity inside the structure, surface temperature and heat flux, simultaneously with exterior and interior environment conditions [10]. The experiment has been carried out on the autoclaved aerated concrete (AAC) and ceramic bricked envelope structure. The brickworks are thermally insulated with the contact system on the basis of graphite expanded polystyrene (EPS). Computed thermal transmittance of the opaque wall part is $U=0.12 \mathrm{~W} / \mathrm{m}^{2} \mathrm{~K}$. Basic material parameters of AAC part of outdoor test cell wall are given in Figure 1 and Table 1, where $\rho$ is the bulk density, $c$ is the specific heat capacity, $\lambda$ is the thermal conductivity. 


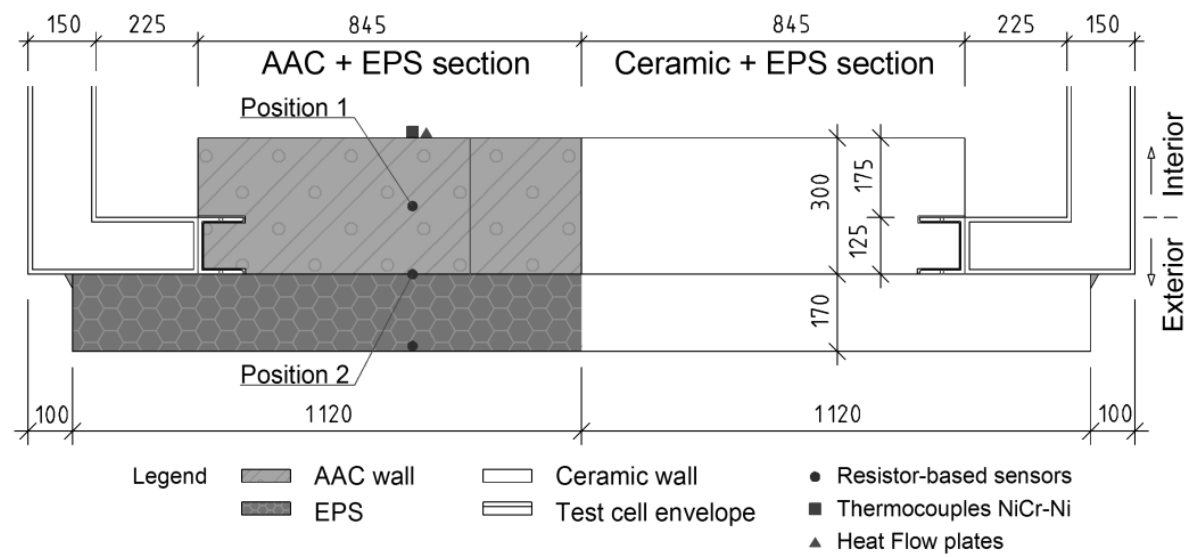

Figure 1: Test cell wall configurations and the sensors positions. Section view of AAC part

Table 1: Composition of the structure (layers are stated in the direction from interior)

\begin{tabular}{l|l|l|l|l|l}
\hline No. & Name of test-wall layer & $\begin{array}{c}d \\
(\mathrm{~m})\end{array}$ & $\begin{array}{c}\lambda_{\mathrm{D}} \\
(\mathrm{W} / \mathrm{m} . \mathrm{K})\end{array}$ & $\begin{array}{c}c \\
(\mathrm{~J} / \mathrm{kg} . \mathrm{K})\end{array}$ & $\begin{array}{c}\rho \\
\left(\mathrm{kg} / \mathrm{m}^{3}\right)\end{array}$ \\
\hline 1 & AAC P2-350 & 0.3000 & 0.1040 & 900.0 & 350.0 \\
\hline 2 & Adhesive PUR foam & 0.0100 & 0.0400 & 800.0 & 35.0 \\
\hline 3 & Graphite polystyrene EPS & 0.1700 & 0.0330 & 920.0 & 16.0 \\
\hline 4 & Adhesive mortar & 0.0020 & 0.8500 & 900.0 & 1300.0 \\
\hline 5 & Primer & - & - & - & - \\
\hline 6 & Silicone plaster & 0.0020 & 0.7000 & 900.0 & 1700.0 \\
\hline
\end{tabular}

\subsection{Boundary conditions}

From the package of measured weather data parameters are chosen exterior and interior air temperature in time period from Nov. $1^{\text {st }}$ till Nov. $6^{\text {th }} 2013$, (in fact the data from Oct. $31^{\text {st }}$ 2013 - for start-up pre-calculation purposes), plotted (Figure 2).

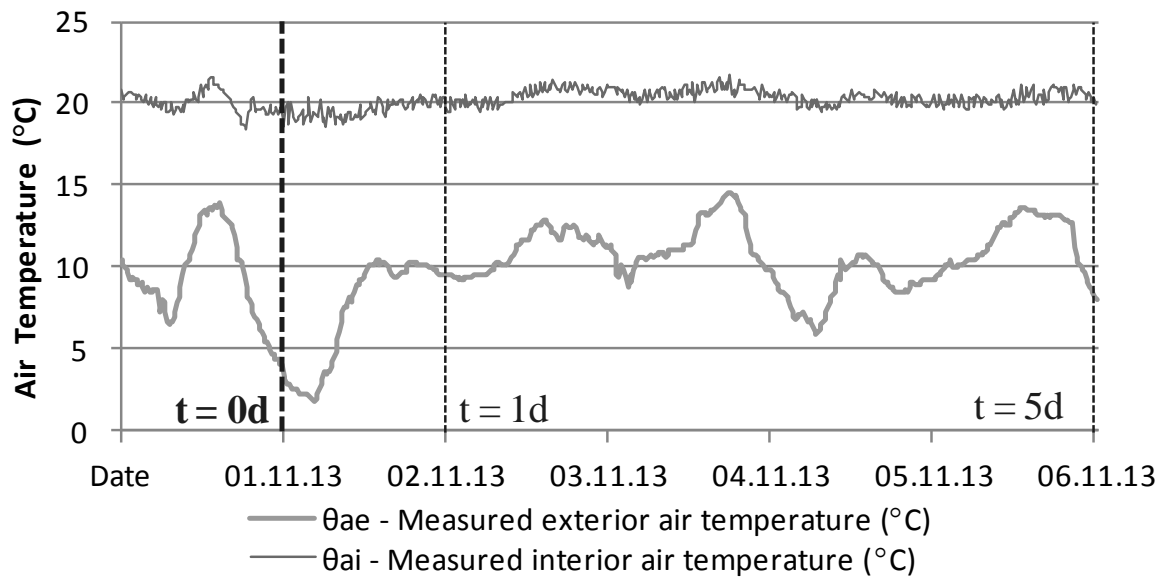

Figure 2: Boundary conditions for initial temperature conditions determination. Measured courses of ambient air temperature during chosen time period 


\section{Results and discussion}

Examined is the effect of initial condition, respectively start-up pre-calculation on the speed of temperature balancing in the real structure. In Figures 3 and 4 there are calculated temperature courses data confronted with measured values in the positions of the test wall (Figure 1). Compared are measured values and numerical analysis results while no start-up pre-calculation and 1 day start-up pre-calculation used. Figures 5, 6, 7, 8 and 9 show temperature profiles comparison across test wall at the start of simulation, after 12 hours, 24 hours, 48 hours and 120 hours (five days). Results analysis is performed in the next chapter.

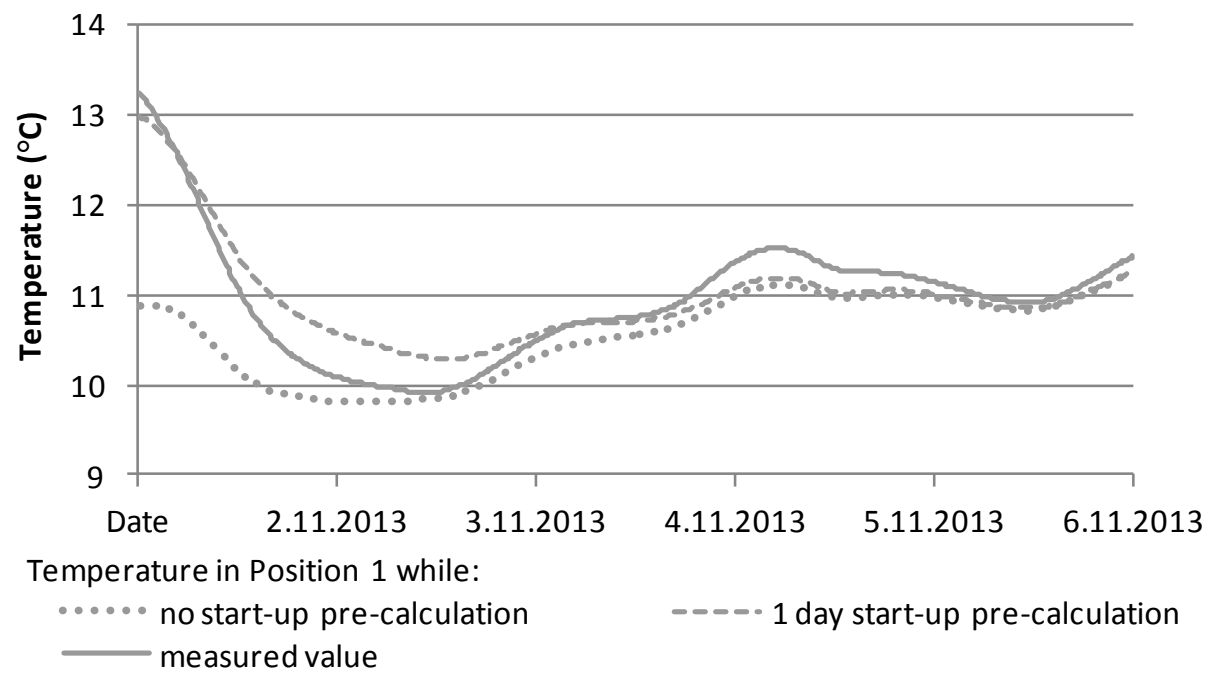

Figure 3: Plotted calculated and measured temperature courses in outdoor test cell's wall in Position 1 during chosen time period. With and without pre-calculation considerations

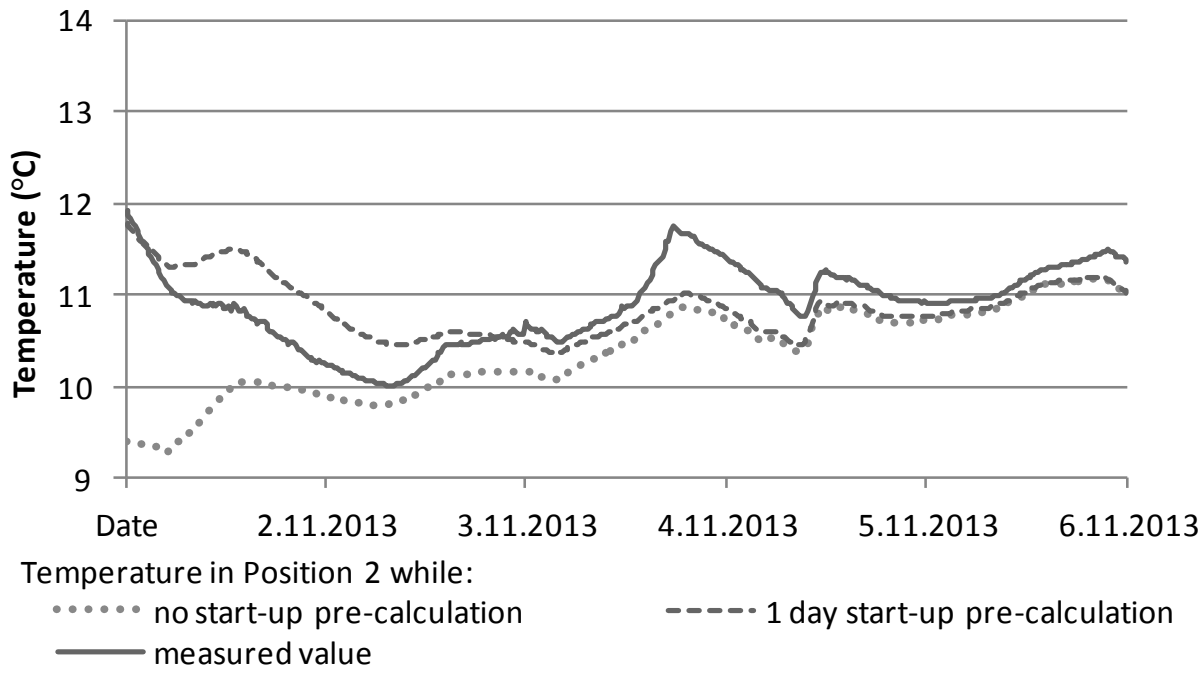

Figure 4: Plotted calculated and measured temperature courses in outdoor test cell wall in Position 2 during chosen time period. With and without pre-calculation considerations 


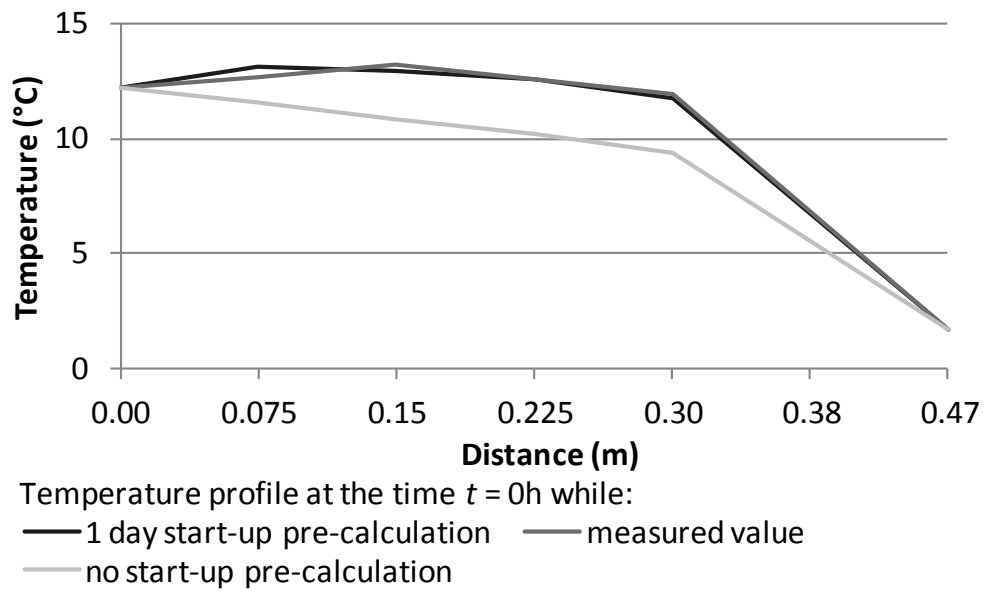

Figure 5: Temperature profile across outdoor test cell wall at the time $t=0 \mathrm{~h}$. With and without precalculation considerations

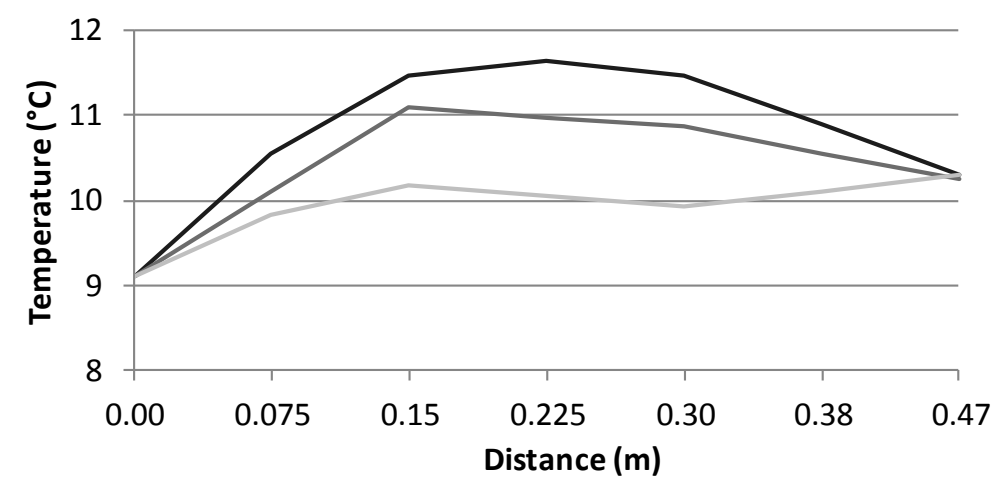

Temperature profile at the time $t=12 \mathrm{~h}$ while:

- 1 day start-up pre-calculation measured value

no start-up pre-calculation

Figure 6: Temperature profile across outdoor test cell wall at the time $t=12 \mathrm{~h}$. With and without precalculation considerations

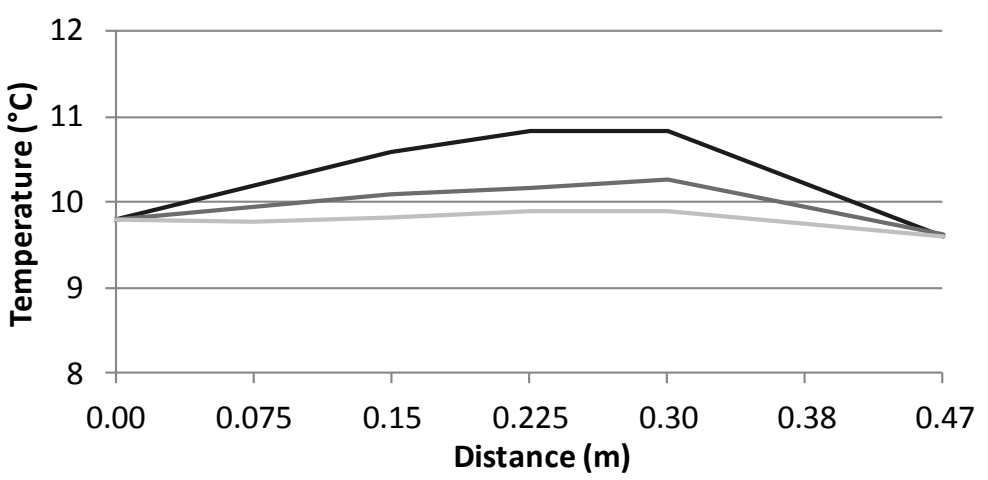

Temperature profile at the time $t=24 \mathrm{~h}$ while:

-1 day start-up pre-calculation - measured value

no start-up pre-calculation

Figure 7: Temperature profile across outdoor test cell wall at the time $t=24 \mathrm{~h}$. With and without pre-calculation considerations 


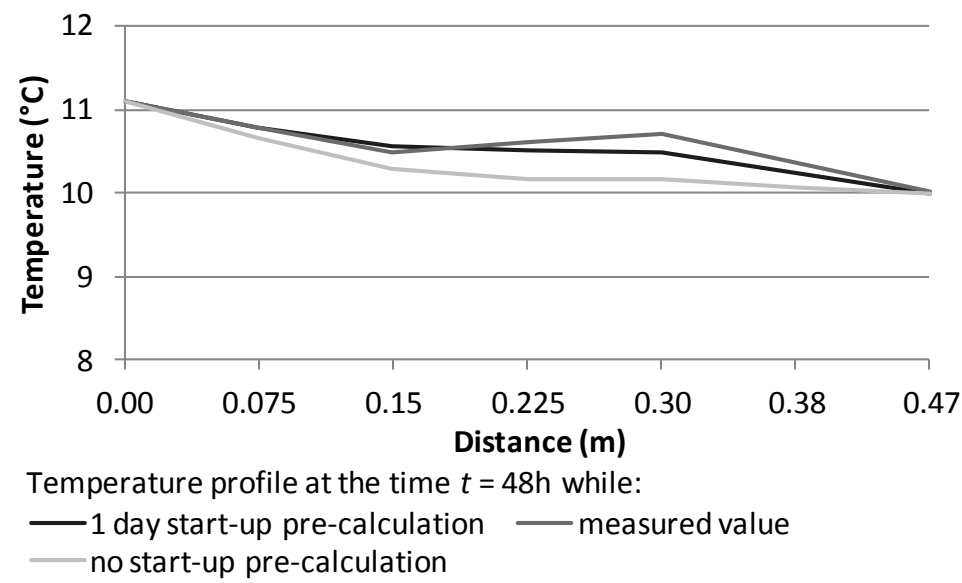

Figure 8: Temperature profile across outdoor test cell wall at the time $t=48 \mathrm{~h}$. With and without precalculation considerations

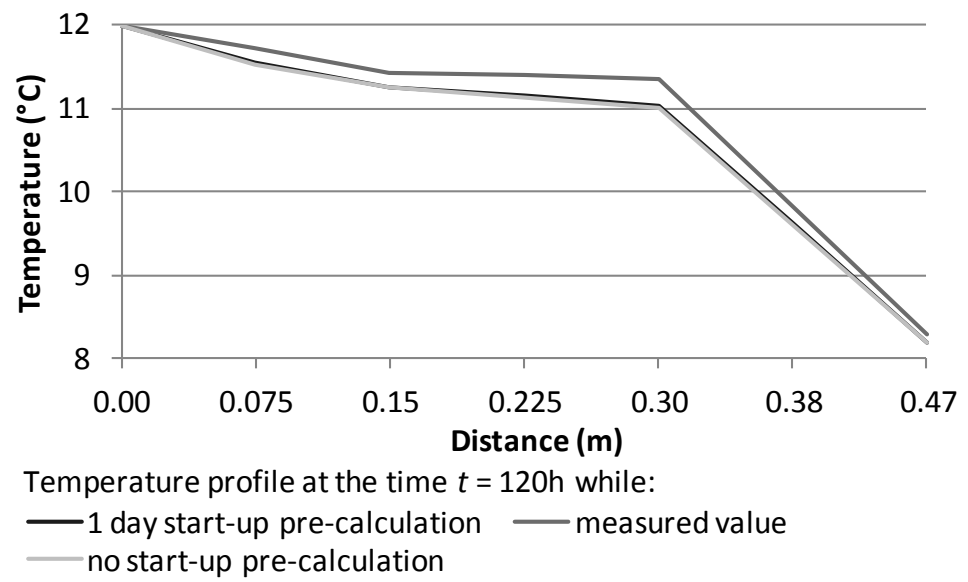

Figure 9: Temperature profile across outdoor test cell wall at the time $t=120 \mathrm{~h}$. With and without precalculation considerations

Having long analysis of temperature fields in non-stationary boundary conditions it possible to omit the initial condition for temperature distribution in the building construction. Omitting the initial condition is disputable when we are speaking about short term analysis lasting less than seven days. The speed of temperature balancing and related validity of the initial condition of the calculation is determined by the composition of the building construction.

Correctly determined initial condition requires information on temperature distribution in the wall's cross section (profile). This information is hardly ever available. Therefore different simplifications are used when building physics analysis needs to be done. Influence of the different way of consideration of the initial condition for the temperature course in the building construction is analysed.

Figure 3 and 4 compare courses of temperatures in two areas of the insulated wall construction according to the schemes displayed on Figure 1. The results of the temperature calculation with and without start-up pre-calculation are compared with measured data. Temperatures calculated using start-up pre-calculation (one day duration) are within first 4 
days in better correlation with measured values. This fact can be better observed on temperature profiles across building construction (Figures 5, 6, 7, 8 and 9). It is possible to observe a positive start-up pre-calculation influence on the accuracy of the calculated values.

Even after 48 hours (Figure 8) there are still differences between start-up and no start-up calculated values of temperature across the construction. After approximately 5 days, balanced temperatures are observed. After that time has elapsed, the influence of the initial condition to results of the simulation is negligible.

Differences between measured and calculated courses are due to neglecting several factors that influence the heat transfer in the building structure. Mainly it is the used simulation tool for analysing the thermal field, without coupling effects of air and moisture to heat transfer, and the effects of moisture content on thermal conductivity and heat capacity of building materials. By using a comprehensive HAM simulation tool and the inclusion of fair moisture and temperature initial condition it is possible to achieve a better agreement of calculated and measured values [11].

\section{Conclusion}

The accuracy of numerical calculations is determined by the correct input data. Initial conditions are often associated with a quantity that persists in structure for a longer period of time for example water content. Entering the initial conditions for temperature is often neglected in the simulation process. The article analyzes the possibilities of entering the initial temperature in transient temperature field simulation. The transient numerical analysis results show that incorrect initial temperature consideration is reflected in balancing of temperature for several days. During these several days there are distorted calculated values. On the other hand, enter the correct initial temperature using start-up pre-calculation showed good compliance with measured temperature values.

\section{Acknowledgements}

This paper was created thanks to the financial support from research project no. 1/0835/14 of Slovak Scientific Grant Agency (VEGA) in collaboration with Slovak Ministry of Education, Science, Research and Sports (MESRS) and Slovak Academy of Sciences (SAS).

\section{References}

[1] Li, Q., Rao, J. \& Fazio, P. (2009). Development of HAM tool for building envelope analysis. Building and Environment. Volume 44. 1065-1673.

[2] Huang, H., Kato, S., Hu, R. \& Ishida, Y. (2011). Development of new indices to assess the contribution of moisture sources to indoor humidity and application to optimization design: Proposal of CRI $(\mathrm{H})$ and a transient simulation for the prediction of indoor humidity. Building and Environment. Volume 46. 1817-1826.

[3] Woloszyn, M. \& Rode, C. (2009). Tools for Performance Simulation of Heat, Air and Moisture Conditions of Whole Buildings. Building Simulation. Volume 1. 5-24.

[4] Katunský, D., Vertal', M., Šimiček, J. \& Zozulák, M. 2014. Numerical analysis and measurement results of a window sill. Advanced Materials Research. Volume 899, 147-150. 
[5] Janssens, A., Roels, S. \& Vandaele, L. (2011). Full scale test facilities for evaluation of energy and hygrothermal performances. Paper presented at International Workshop. Brussels, Belgium.

[6] Baker, P.H. \& van Dijk, H.A.L. (2008). PASLINK and dynamic outdoor testing of building components. Building and Environment. Volume 43, 143-151.

[7] Strachan, P.A. \& Vandaele, L. (2008). Case studies of outdoor testing and analysis of building components. Building and Environment. Volume 43, 129-142.

[8] Fraunhofer Institute for Building Physics WUFI (2011, Jun). Creating Weather Files. Retrieved Jun 16, 2011, from http://www.wufi.de/frame_en_wetterdaten.html

[9] Physibel. (2013). User manual for Physibel [computer software]. Maldegem, Belgium: Physibel.

[10] Katunský, D., Vertal', M. \& Zozulák, M. (2014). Experimentally Measured Boundary and Initial Conditions for Simulations. Advanced Materials Research. Volume 1041, 293-296.

[11] Vertal', M., Katunský, D. \& Buriková, D. (2012). Water profile as initial condition for HAM analysis. Thermal Protection of Buildings. Volume 5, 3-6. (In Slovak). 